Ann. Biol. anim. Bioch. Biophys., Ig68, 8 (3), 44I-444.

\author{
NOTE, TECHNIQUE
}

\title{
UNE TECHNIQUE SIMPLE DE PONCTION CARDIAQUE CHEZ LA TRUITE
}

\author{
M. DORSON \\ avec la collaboration technique de Annie Dubors et Yvette BAUdrIER \\ Station d'hydrobiologie continentale, 14, avenue de Saint-Mandé Paris 12e, \\ Institut national de la Recherche agronomique
}

Cette technique a été mise au point en vue d'études sérologiques et immunologiques chez la truite arc-en-ciel (Salmo gairdneri).

Plusieurs techniques déjà expérimentées avec succès, se sont révélées d'application délicate aux poissons de la taille considérée $(25 \mathrm{~cm})$. Aussi avons-nous mis au point une technique de ponction intra-atriale.
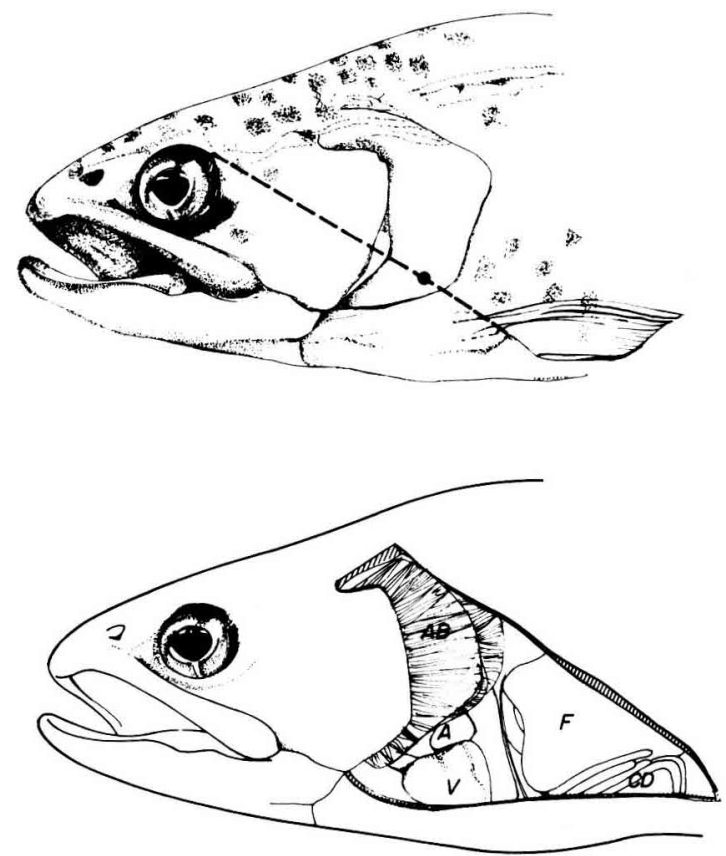

Fig. I. - Schémas réalisés d'après deux photos de la même truite, d la même échelle, avant et après dissection, permettant le repérage de l'atrium : $\mathrm{A}$ : atrium, $\mathrm{AB}:$ arcs branchiaux, $\mathrm{CD}:$ cæca digestifs. $\mathrm{F}:$ foie, $\mathrm{V}$ : ventricule. 
Les poissons (truites " portion " de 25 à $27 \mathrm{~cm}, 25^{\circ} \mathrm{g}$ environ) sont anesthésiés à la métacaïne (M. S. 222 Sandoz) Ioo p.p.m., et maintenus à plat, sur le flanc, à l'aide d'un linge. D'une main l'opérateur tient la tête de l'animal et soulève l'opercule à l'aide d'un doigt. De l'autre, il tient la seringue et enfonce verticalement l'aiguille héparinée $(20 / 6$ biseau + de $\mathrm{I} \mathrm{mm})$ à travers la paroi de la cavité branchiale, à l'intersection d'une ligne fictive joignant le sommet de l'orbite à la base de l'insertion de la nageoire pectorale et des feuillets branchiaux du $4^{e}$ arc. Il n'est pas nécessaire d'enfoncer l'aiguille profondément pour atteindre l'atrium : généralement $2 \mathrm{~mm}$.

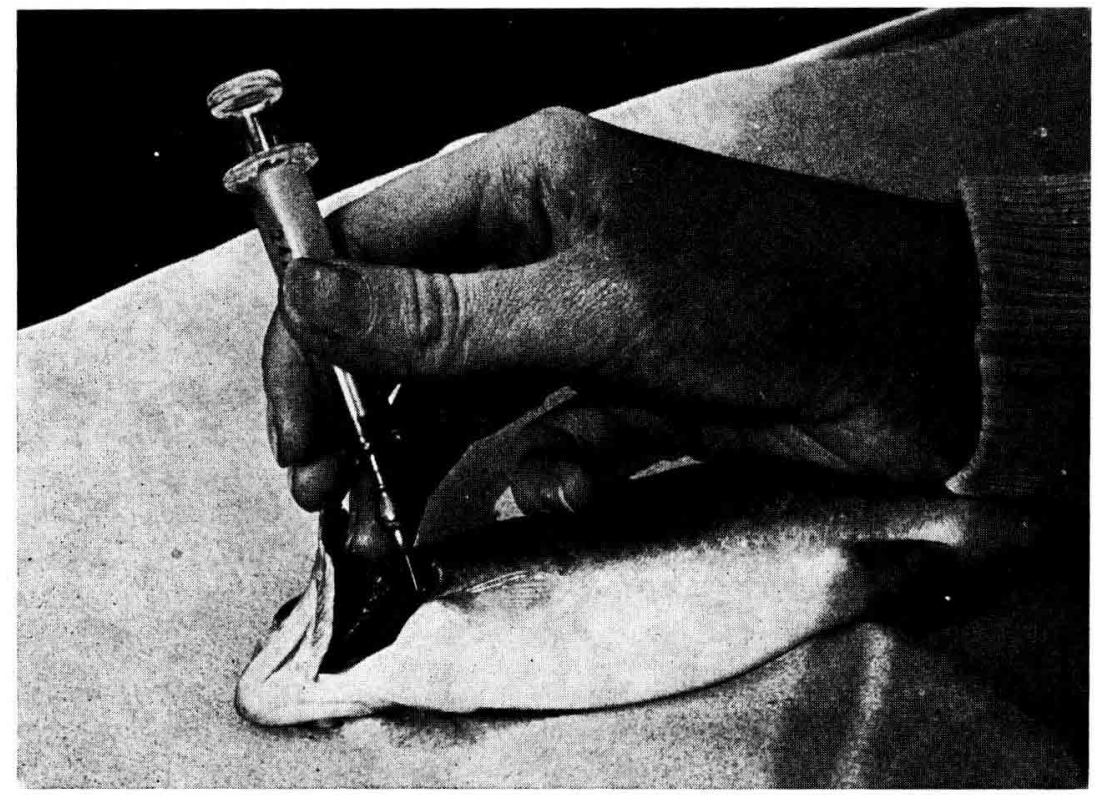

FIG. 2. - Photographie montrant la position de la seringue lors de la prise de sang

\section{RÉSULTATS}

\begin{tabular}{|c|c|c|c|c|c|}
\hline \multicolumn{3}{|c|}{ Lot $1: 15$ truites } & \multicolumn{3}{|c|}{ Lot $2: 15$ truites } \\
\hline Date de ponction & $\begin{array}{l}\text { Sang } \\
\left(\mathrm{cm}^{3}\right)\end{array}$ & Mortalité & Date de ponction & $\begin{array}{l}\text { Sang } \\
\left(\mathrm{cm}^{3}\right)\end{array}$ & Mortalité \\
\hline $24-10-67$ & 0,2 & 0 & $26-10-67$ & 0,2 & 0 \\
\hline $31-10-67 \ldots \ldots \ldots \ldots$ & 0,5 & 0 & $2-11-67$ & 0,5 & 0 \\
\hline $7-11-67 \ldots \ldots \ldots \ldots$ & 0,5 & 0 & $9-11-67 \ldots \ldots \ldots \ldots$ & 0,5 & 0 \\
\hline $14-11-67 \ldots \ldots \ldots \ldots \ldots$ & 1 & 1 & $16-11-67 \ldots$ & 1 & 1 \\
\hline $21-11-67 \ldots \ldots \ldots \ldots \ldots$ & 1 & $\mathbf{0}$ & 23-11-67 $\ldots$. & 1 & 0 \\
\hline $28-11-67 \ldots \ldots \ldots \ldots \ldots$ & 2 & 0 & $30-11-67 \ldots \ldots \ldots \ldots$ & 2 & 0 \\
\hline & & & & & \\
\hline
\end{tabular}

Les truites du lot $\mathrm{n}^{0}$ I ont été sacrifiées le 8 décembre 1967 pour contrôler l'existence de lésions éventuelles. L'observation sous loupe binoculaire des cœurs maintenus dans du sérum physiologique n'a révélé aucune lésion microscopique. 
Le contrôle histologique (fixation au liquide de Bouin, coloration à l'hémalun-éosine-safran) n'a montré l'existence d'aucune lésion microscopique.

\section{DISCUSSION}

La technique décrite par Schiffman (1959) utilisant l'aorte dorsale antérieure par voie palatine s'applique plutôt à des espèces à grande bouche, et à des poissons d'une certaine taille $\left(3^{\circ} \mathrm{cm}\right.$ au moins). Il en est de même pour la technique de SMITH et al. (1964), qui consiste à placer à demeure une canule dans l'aorte dorsale antérieure et qui ne répond pas à nos impératifs de simplicité. AMES et al. (rg66) utilisent l'artère branchiale efférente, ce qui pose des problèmes pour les petits sujets. MolNar (I960) effectue des ponctions dans le ventricule par voie médio-ventrale : l'aiguille doit donc traverser la paroi musculaire avant d'atteindre le cœur, d'où des risques d'obstruction avec les aiguilles de faible diamètre employées pour les petits poissons. Les mêmes critiques s'appliquent aux ponctions utilisant un tube enfoncé dans le pédoncule caudal (STEUCKE et al., ig67). Des incisions dans le sinus veineux ont été réalisées par le même auteur, mais leur répétition semble délicate.

Comparée à ces diverses techniques, celle que nous avons mise au point présente les avantages suivants :

- elle est simple et ne nécessite qu'un matériel réduit ;

- elle est rapide, évite une manipulation prolongée du poisson et peut s'effectuer n'importe où (travail de terrain);

- elle est peu traumatisante, et se répète facilement, surtout si on alterne les ponctions, tantôt à droite, tantôt à gauche ;

- l'aiguille ne traverse que la membrane de la cavité branchiale avant d'atteindre l'atrium : il n'y a aucun risque d'obstruction.

Nous avons envisagé l'application aux cyprinidés et effectué des essais sur le Gardon(Rutilus rutilus), le Chevesne (Leuciscus cephalus) et le Hotu, (Chondrostoma nasus). Sans nous étendre sur ces essais, signalons que la position de l'aiguille est à déterminer avec précision pour chaque espèce et que, pour le Hotu, il est pratiquement impossible d'effectuer la ponction dans l'atrium, celui-ci étant petit et masqué par le ventricule : on peut cependant effectuer par cette technique une ponction intraventriculaire.

Reçu pour publication en mars 1968.

\section{SUMMARY}

\section{A SIMPLE TECHNIQUE FOR OBTAINING TROUT BLOOD BY CARDIAC PUNCTURE}

The puncture is made through the branchial membrane into the atrium, by means of a syringe, at the point of intersection of a virtual line joining the top of the orbit to the basement of the pectoral fin, with the 4 th gill arch. fishes.

This technique is simple, easily repeatable for it causes no trauma, applicable to short-sized 


\section{RÉFÉRENCES BIBLIOGRAPHIQUES}

Ames W. E., Condie R. M., Pollara B., r966. A rnethod for intravascular injection and bleeding of fishes. Trans. Am. Fish. Sac., 95, 31 7-318.

Conte F. P., Wagner H. M., Harris T. G., I963. Measurements of blood volume in the fish (Salmo gairdneri gairdneri). Amer. J. Physiol., 205, 533-540.

MolNaR G., rg6o. Methode der Blutentnahme für haematologische Untersuchungen bei Fischen. Z. Fish. Hilfswissensch., Dtsch., 9, IоI-го6.

Perkins F. E., r957. A field technique for obtaining samples of fish blood serum. Progve Fish. Cult. 19, 144 .

Schiffman R. H., I959. Method for repeated sampling of trout blood. Progve Fish. Cult., 21, I5I-I53.

Smirt S. L., Bell G. R., 1964. A technique for prolonged blood sampling in free-swimming salmon. J. Fish. Res. Bd Can., 21, 71 I-7I7.

Smith L. S., Ig66. Blood volumes of three salmonids. J. Fish. Res., Bd Can., 23, I439-I 446.

Steucke E. W., SchötTGer R. A., I967. Comparison of three methods of sampling trout blood for measurements of hematocrit. Progve Fish Cult., 29, 98-104. 